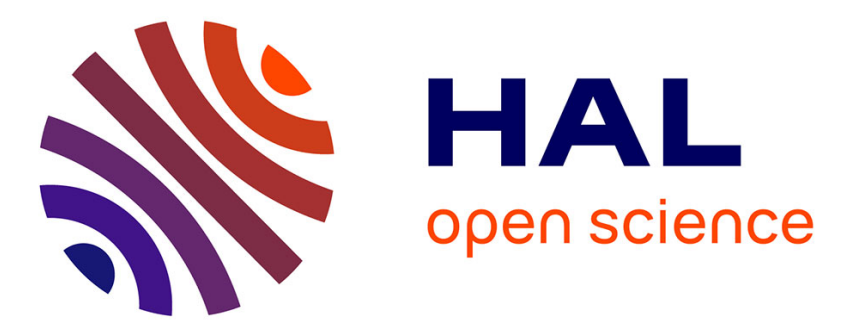

\title{
Higher order differential attacks on iterated block ciphers using almost bent round functions
}

\author{
Anne Canteaut, Marion Videau
}

\section{To cite this version:}

Anne Canteaut, Marion Videau. Higher order differential attacks on iterated block ciphers using almost bent round functions. ISIT 2002 : IEEE International Symposium on Information Theory, Jun 2002, Lausanne, Switzerland. pp.209, 10.1109/ISIT.2002.1023481 . hal-00675354

\section{HAL Id: hal-00675354 https://hal.inria.fr/hal-00675354}

Submitted on 1 Mar 2012

HAL is a multi-disciplinary open access archive for the deposit and dissemination of scientific research documents, whether they are published or not. The documents may come from teaching and research institutions in France or abroad, or from public or private research centers.
L'archive ouverte pluridisciplinaire HAL, est destinée au dépôt et à la diffusion de documents scientifiques de niveau recherche, publiés ou non, émanant des établissements d'enseignement et de recherche français ou étrangers, des laboratoires publics ou privés. 


\title{
Higher order differential attacks on iterated block ciphers using almost bent round functions
}

\author{
Anne Canteaut and Marion Videau \\ INRIA projet CODES, \\ BP 105, 78153 Le Chesnay Cedex, FRANCE \\ e-mail: \{Anne.Canteaut, Marion.Videau\}@inria.fr
}

\begin{abstract}
We show that the use of a round function whose Walsh coefficients are divisible by a high power of 2 may lead to a higher order differential attack.
\end{abstract}

\section{Higher ORDER DIFFERENTIAL ATtACKS}

We consider an $r$-round iterated block cipher with block size $n: F_{k_{r}} \circ F_{k_{r-1}} \circ \ldots \circ F_{k_{1}}$ where the round function $F_{k}$ is a permutation of $\mathbf{F}_{2}^{n}$ for any $k$. Such a cipher is vulnerable to linear cryptanalysis if there exists $(\alpha, \beta), \alpha \neq 0$ such that $\alpha \cdot F_{k}(x)+\beta \cdot x$ takes the same value for most values of $x \in \mathbf{F}_{2}^{n}$. This property is related to the Walsh spectrum of $F_{k}$.

For any $\alpha \in \mathbf{F}_{2}^{n}, \varphi_{\alpha}$ denotes the linear function $x \mapsto \alpha \cdot x$ where "." is the usual dot product. The Walsh spectrum of a function $F$ from $\mathbf{F}_{2}^{n}$ into $\mathbf{F}_{2}^{n}$ is the multiset

$$
\left\{\mathcal{F}\left(\varphi_{\alpha} \circ F+\varphi_{\beta}\right)=\sum_{x \in \mathbf{F}_{2}^{n}}(-1)^{\alpha \cdot F(x)+\beta \cdot x}, \alpha \in \mathbf{F}_{2}^{n} \backslash\{0\}, \beta \in \mathbf{F}_{2}^{n}\right\} .
$$

The nonlinearity of $F$ is the Hamming distance between all $\varphi_{\alpha} \circ F$ and the set of affine functions. It is equal to

$2^{n-1}-\frac{1}{2} \mathcal{L}(F) \quad$ where $\quad \mathcal{L}(F)=\max _{\alpha \in \mathbf{F}_{2}^{n}} \max _{\beta \in \mathbf{F}_{2}^{n}}\left|\mathcal{F}\left(\varphi_{\alpha} \circ F+\varphi_{\beta}\right)\right|$.

The minimum value for $\mathcal{L}(F)$ is $2^{\frac{n+1}{2}}$ and the functions achieving this value are called almost bent functions. They provide a high resistance to both linear and differential attacks [2].

The derivative of a function $F$ over $\mathbf{F}_{2}^{n}$ with respect to a linear subspace $V$ is defined by $D_{V} F(x)=\sum_{v \in V} F(x+v)$ where the sum is an addition modulo 2. Suppose that for any round keys, the reduced cipher, i.e., the function $G=$ $F_{k_{r-1}} \circ \ldots \circ F_{k_{1}}$, has degree $d$ (the degree of $G$ is the maximum degree of its Boolean components). Then, for any $(d+1)$ dimensional subspace $V$, we have $\sum_{v \in V} G(x+v)=0$ for all $x \in \mathbf{F}_{2}^{n}$. It leads to a differential attack of order $(d+1)[4]$ :

1. Select a random plaintext $x_{0} \in \mathbf{F}_{2}^{n}$ and get the ciphertexts $c_{v}$ corresponding to all plaintexts $x_{0}+v, v \in V$.

2. For each candidate for $k_{r}$, compute $\sum_{x \in V} F_{k_{r}}^{-1}\left(c_{v}\right)$.

The key $k_{r}$ for which this sum vanishes is the correct lastround key with a high probability. But, the bound $\operatorname{deg}(G) \leq$ $(\operatorname{deg}(F))^{r-1}$ only enables to apply the attack to the ciphers with low degree round functions.

\section{Divisibility of the WAlsh SPECTRUm AND DEGREE OF A COMPOSED FUNCTION}

The trivial bound $\operatorname{deg}(F \circ F) \leq \operatorname{deg}(F)^{2}$ can be improved when the values occurring in the Walsh spectrum of $F$ are divisible by a high power of 2. By using a relation between the Walsh coefficients of the sum of some Boolean functions and the Walsh coefficients of their product, we can prove
Theorem 1 Let $F$ be a function from $\mathbf{F}_{2}^{n}$ into $\mathbf{F}_{2}^{n}$ such that all values occurring in its Walsh spectrum are divisible by $2^{\ell}$. Then, for any function $F^{\prime}$ from $\mathbf{F}_{2}^{n}$ into $\mathbf{F}_{2}^{n}$, we have

$$
\operatorname{deg}\left(F^{\prime} \circ F\right) \leq n-\ell+\operatorname{deg}\left(F^{\prime}\right) .
$$

This result is of great interest when $F$ is almost bent since the Walsh coefficients of any almost bent function over $\mathbf{F}_{2}^{n}$ are divisible by $2^{\frac{n+1}{2}}[2]$. It leads to a new attack on any 5 -round Feistel cipher using a highly nonlinear substitution function. In a Feistel cipher with block size $2 n$, the round function is defined by $(L, R) \mapsto\left(R, L+S_{k}(R)\right)$ where $L$ and $R$ are the left and right halves of the input and $S_{k}$ is a function over $\mathbf{F}_{2}^{n}$ called the substitution function. The right part of the output of the third round, $R_{3}$, can be derived from the ciphertext $\left(L_{5}, R_{5}\right)$ and from $k_{5}: R_{3}=R_{5}+S_{k_{5}}\left(L_{5}\right)$. Moreover, when we consider any plaintext $\left(x, c_{0}\right)$ whose right part is a constant, we have $R_{3}(x)=x+c_{1}+S_{K_{3}}\left(c_{0}+S_{K_{2}}\left(x+c_{1}\right)\right)$. When the confusion function $S$ is almost bent, Theorem 1 implies that $\operatorname{deg}\left(R_{3}\right) \leq \frac{n-1}{2}+\operatorname{deg}(S)$. Thus, we have exhibited a new differential attack of order $\delta=\min \left(\left(\operatorname{deg}(S)^{2}+1, \frac{n+1}{2}+\operatorname{deg}(S)\right)\right.$. Since the degree of an almost bent function cannot exceed $(n+1) / 2$, this attack is feasible except for almost bent functions of maximal degree. But, it can be improved and performed for any almost bent functions when the round key is inserted by addition. It also applies when $S_{k}$ is a permutation of an even number of variables which has the highest known nonlinearity. Except the inverse function, all known permutations $S$ achieving $\mathcal{L}(S)=2^{n / 2+1}$ are such that all their Walsh coefficients are divisible by either $2^{n / 2}$ or $2^{n / 2+1}[3]$.

Theorem 1 also provides an explanation and a generalization of a 7-order differential attack on a reduced version of MISTY1 [6, 1]. This weakness originates from the use of almost bent substitution boxes in the cipher.

\section{REFERENCES}

[1] S. Babbage and L. Frisch. On MISTY1 higher order differential cryptanalysis. In ICISC 2000, LNCS 2015, pp. 22-36. SpringerVerlag, 2000.

[2] F. Chabaud and S. Vaudenay. Links between differential and linear cryptanalysis. In EUROCRYPT'94, LNCS 950, pp. 356365. Springer-Verlag, 1995.

[3] H. Dobbertin. One-to-one highly nonlinear power functions on $G F\left(2^{n}\right)$. Appl. Algebra Engrg. Comm. Comput., 9(2):139-152, 1998 .

[4] L. R. Knudsen. Truncated and higher order differentials. In FSE'94, LNCS 1008, pp. 196-211. Springer-Verlag, 1995.

[5] X. Lai. Higher order derivatives and differential cryptanalysis. In Symposium on Communication, Coding and Cryptography in honor of J. Massey on the occasion of his 60'th birthday, 1994.

[6] H. Tanaka, K. Hisamatsu, and T. Kaneko. Strength of MISTY1 without FL function for higher order differential attack. In $A A E C C$-13, LNCS 1719, pp. 221-230. Springer-Verlag, 1999. 